\title{
A scalable, optically-driven, high-voltage switch for remote MEMS device operation fabricated with a standard CMOS process
}

\author{
Isao Mori, Yuki Okamoto, and Yoshio Mita ${ }^{\text {a) }}$ \\ Department of Electrical Engineering and Information Systems, \\ the University of Tokyo, \\ 7-3-1 Hongo, Bunkyo-ku, Tokyo 113-8656, Japan \\ a)mems@if.t.u-tokyo.ac.jp
}

\begin{abstract}
We propose a high-voltage switch driven by wavelength-modulated light, which is suitable for remote MEMS (especially electrostatic actuators) device operation. It is fabricated on an SOI wafer and consists of series-connected n-MOSFETs whose substrates are mesa-isolated, allowing the switch to endure a high voltage. The fabrication process requires no modification in a standard CMOS process itself, which is easy for foundries to accept. In addition, by using wavelength-modulated light instead of electric circuits for the control of the switch, it achieves a simple, scalable, small switching circuit regardless of the switching voltage. Fabrication and operation of $50 \mathrm{~V}$ switch using $5 \mathrm{~V}$ standard CMOS technology is reported in this paper.
\end{abstract}

Keywords: high-voltage switch, standard CMOS process, MEMS, wavelength-modulated light

Classification: Micro- or nano-electromechanical systems

\section{References}

[1] J. M. Kahn, et al:: "Next century challenges: mobile networking for "smart dust"," Proc. MobiCom'99 (1999) 271 (DOI: 10.1145/313451.313558).

[2] B. A. Warneke, et al:: "An autonomous $16 \mathrm{~mm}^{3}$ solar-powered node for distributed wireless sensor networks," Proc. IEEE Sensors 2002 (2002) 1510 (DOI: 10.1109/ICSENS.2002.1037346).

[3] O. Fuchiwaki and H. Aoyama: "Flexible micro-processing by multiple miniature robots in SEM vacuum chamber," Proc. 11th Int. Symp. Micromechatronics and Human Science (2000) 145 (DOI: 10.1109/MHS.2000. 903304).

[4] M. Nakada, et al.: "Optical coherence tomography by all-optical MEMS fiber endoscope," IEICE Electron. Express 7 (2010) 428 (DOI: 10.1587/elex.7.428).

[5] R. N. Simons, et al.: "RF telemetry system for an implantable bio-MEMS sensor," IEEE MTT-S Int. Microw. Symp. Dig. 3 (2004) 1433 (DOI: 10.1109/ MWSYM.2004.1338841). 
[6] P. Cong, et al.: "A wireless and batteryless 10-bit implantable blood pressure sensing microsystem with adaptive RF powering for real-time laboratory mice monitoring," IEEE J. Solid-State Circuits 44 (2009) 3631 (DOI: 10.1109/JSSC. 2009.2035551).

[7] E. Y. Chow, et al.: "Fully wireless implantable cardiovascular pressure monitor integrated with a medical stent," IEEE Trans. Biomed. Eng. 57 (2010) 1487 (DOI: 10.1109/TBME.2010.2041058).

[8] J. D. Plummer and J. D. Meindl: "A monolithic 200-V CMOS analog switch," IEEE J. Solid-State Circuits 11 (1976) 809 (DOI: 10.1109/JSSC.1976. 1050822).

[9] M. Declercq, et al.: "Design and optimization of high-voltage CMOS devices compatible with a standard 5 V CMOS technology," Proc. IEEE 1993 Custom Integrated Circuits Conf. (1993) 24.6.1 (DOI: 10.1109/CICC.1993.590766).

[10] S. Morishita, et al:: "A bridge-connected isolated silicon islands postprocessing method for fine-grain-integrated $\pm 10 \mathrm{~V}$-operating CMOS-MEMS by standard 5 V CMOS process technology," Proc. MME2010 (2010) 48.

[11] Y. Okamoto, et al.: "Demonstration of 0-30 V comb-drive MEMS actuator by integrated switching circuit with post-mesa-isolated standard $5 \mathrm{~V}$ CMOS transistors," DTIP2016 (2016) 1 (DOI: 10.1109/DTIP.2016.7514893).

[12] I. Mori: "A robust on-chip-integrated high-voltage photovoltaic cell array and application to high-speed remote optical operation of MEMS actuators," Ph.D. Dissertation, The University of Tokyo (2016).

[13] I. Mori, et al.: "Discharging-phototransistor-integrated high-voltage Si photovoltaic cells for fast driving demonstration of an electrostatic MEMS actuator by wavelength modulation," Jpn. J. Appl. Phys. 55 (2016) 04EF12 (DOI: 10. 7567/JJAP.55.04EF12).

\section{Introduction}

The development of microelectromechanical systems (MEMS) technologies allows remotely working devices [1, 2, 3, 4, 5, 6, 7]. Since MEMS devices, especially electrostatic actuators, require high-voltage switching (e.g. $30 \mathrm{~V}$ ) in the operation, high-voltage integrated control circuits are necessary to make them truly effective. However, conventional high-voltage technologies have issues such as modification in a standard CMOS process [8] or slow switching [9].

The authors' group previously proposed a mesa-isolated CMOS fast witch on a silicon-on-insulator (SOI) wafer [10, 11]. This method isolates the substrate of each MOSFET by post-process etching, which allows the transistors to endure a high voltage without modifying a standard CMOS process itself. However, it requires multiple voltage sources (e.g. a $50 \mathrm{~V}$ switch requires $5,10, \ldots, 50 \mathrm{~V}$ ) and tedious voltage level shifters for them, which results in a complex circuit and a large area.

In this paper, we propose a high-voltage switch for remote MEMS device operation driven by wavelength-modulated light [12], which employs the method of circuit function switching that we previously proposed [13]. By controlling the switch with light instead of electricity, we eliminate complex voltage level shifters, which achieves high-voltage switching with small number of transistors that are 


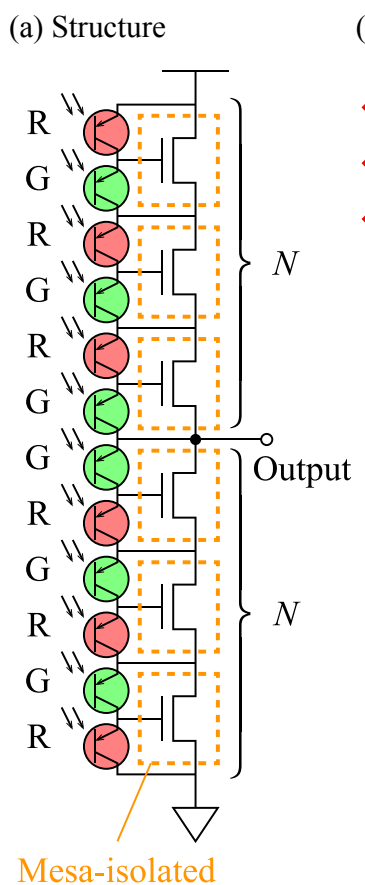

(b) ON state
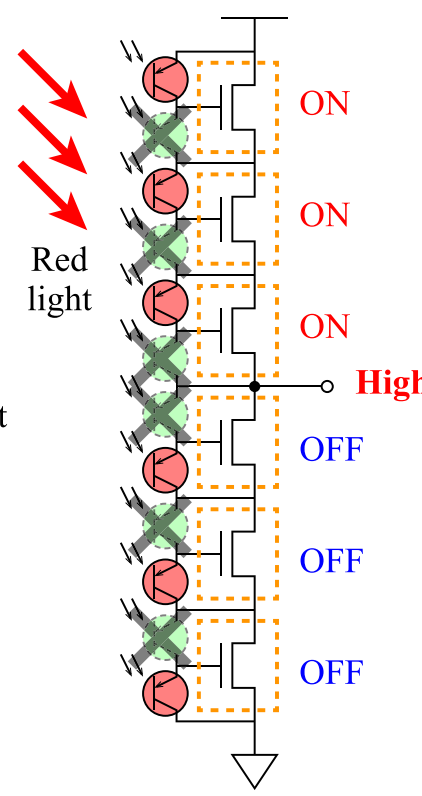

(c) OFF state

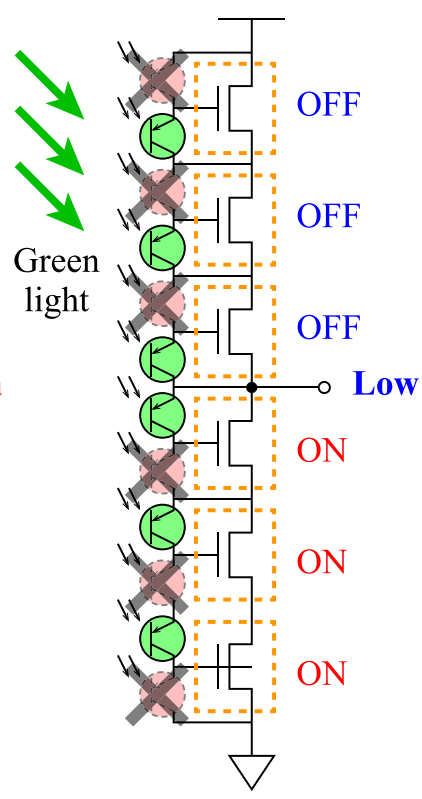

Fig. 1. Operation principle of the proposed high-voltage switch driven by wavelength-modulated light

\section{Proposed circuit}

Fig. 1 explains the concept of the proposed high-voltage switch. It has a similar structure to a cascaded CMOS inverter, but the transistors are all n-MOSFET and the number of the transistor pairs $(N)$ is $V_{\mathrm{dd}} / 5$, where $V_{\mathrm{dd}}[\mathrm{V}]$ and "5" [V] are the switching voltage and the supply voltage of the CMOS technology, respectively. The transistors are all mesa-isolated, which makes it possible to apply independent voltages to the body of each transistor. This large number of transistors and the mesa-isolation allow the switch out of low voltage transistors to endure a high voltage.

The key to the operation of this high-voltage switch is the phototransistors connected to the gate of each transistor. As shown in Fig. 1, they are colored in red and green with lacquer to ensure the following complemental on-off operation: (1) if the switch is illuminated with red light, the transistors of the high $\left(V_{\mathrm{pp}}\right)$ side turn on and those in the low (ground) part turn off, which makes the output of the switch high, and (2) under green light, on the other hand, the low side turns on and the high side turns off, which makes the output low.

In this method, the switch employs standard n-MOSFETs, allowing low onresistance, and the mesa-isolation of the n-MOSFETs are performed in a way compatible with a standard CMOS process. In addition, it requires only small phototransistors instead of complex sub-switches for switching the n-MOSFETs, which allows the relatively small area of the switch.

\section{Fabrication and measurements}

For the demonstration of the concept, a $50 \mathrm{~V}$ high-voltage switch, which has 10 


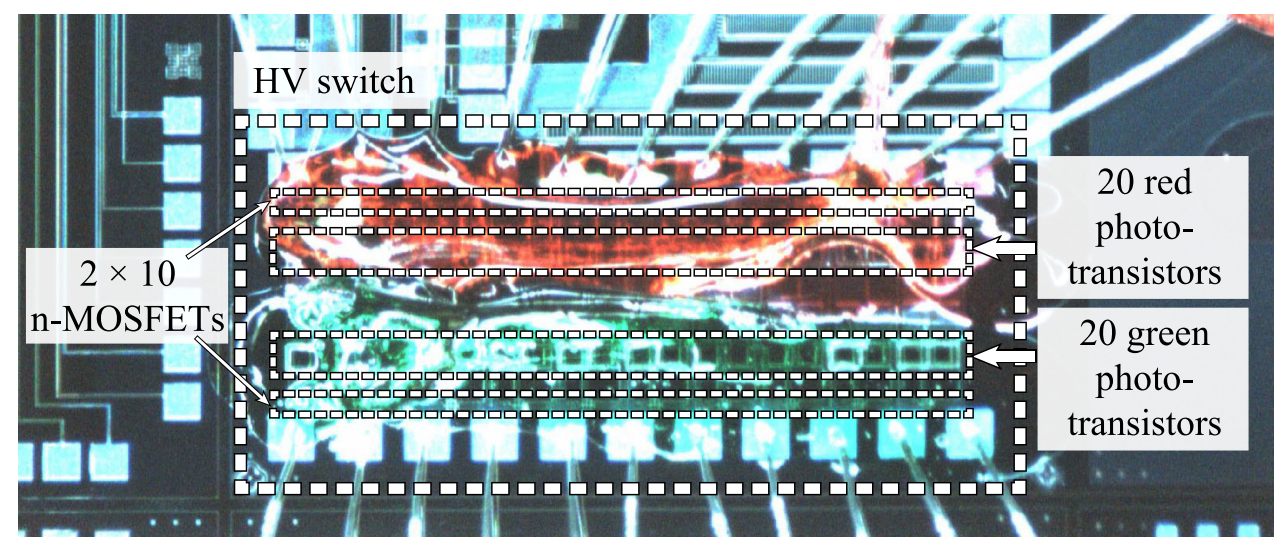

Fig. 2. Fabricated chip of the high-voltage switch

drain and the source of each transistor had pads as taps so that the subsets of the switch can be also measured. The length and the width of the MOSFET were $0.6 \mu \mathrm{m}$ and $10 \mu \mathrm{m}$, respectively, and the area of the phototransistor was $4719.65 \mu \mathrm{m}^{2}$. Fig. 2 shows one of the devices fabricated in the method explained in the authors' previous work [13]. That is, an SOI wafer delivered from the CMOS foundry was post-processed to mesa-isolate the n-MOSFETs and the phototransistors with anisotropic and isotropic plasma etching, followed by the painting of lacquer in red and green.

The measurements of the fabricated device were conducted with a semiconductor parameter analyzer. The light source was a floodlight which has red and green LEDs.

First, the drain current characteristics of a single n-MOSFET block were measured (Fig. 3). The $x$-axis of (a) is the current applied to the floodlight LEDs $\left(I_{\mathrm{LED}}\right)$, to which the intensity of the light is proportional. The drain current of an n-MOSFET successfully changed by alternating the color of light as long as the current flowing through LEDs was $7 \mathrm{~mA}$ or larger. The on-current was $4.11 \mathrm{~mA}$, and the off-current (leak current) was $42.6 \mathrm{nA}$. The characteristics of the n-MOSFET's drain current $\left(I_{\mathrm{d}}\right)$ against its drain voltage $\left(V_{\mathrm{ds}}\right)$ was quite different from that of an ordinary n-MOSFET. This is because the structure of an $\mathrm{n}$-MOSFET block is in a diode connection.

Next, the measurement results of the $50 \mathrm{~V}$ switch are shown in Fig. 4. As shown in Fig. 4(a), the $50 \mathrm{~V}$ switch worked as long as the LED current was $5 \mathrm{~mA}$ or larger. In this measurement, $I_{\mathrm{d}}$ was $247 \mathrm{nA}$ in the ON state and $29.7 \mathrm{nA}$ in the OFF state. Fig. 4(b) shows the output voltage characteristics of the switch against various numbers of n-MOSFET pairs $(N)$. The solid line is $V_{\mathrm{dd}}$, which is the output voltage of the ideal switch in the ON state. The measured output voltages were close to $V_{\mathrm{dd}}$ in the $\mathrm{ON}$ state and to $0 \mathrm{~V}$ in the OFF state, though there were gaps between the ideal output voltages and the measured output voltages. The average gaps in the ON and OFF states were $(N \times 0.406) \mathrm{V}$ and $(N \times 0.424) \mathrm{V}$, respectively.

\section{Discussion}

๑ IEICE 2017

DOI: 10.1587/elex.14.20161174 Received November 26, 2016 Accepted December 21, 2016 Publicized January 16, 2017 Copyedited February 10, 2017
The measurement in the Fig. 4 shows three things: (1) turning on and off the circuit requires minimum amount of light $\left(I_{\text {LED }}>5 \mathrm{~mA}\right)$, (2) 'high-side' voltage gets its 

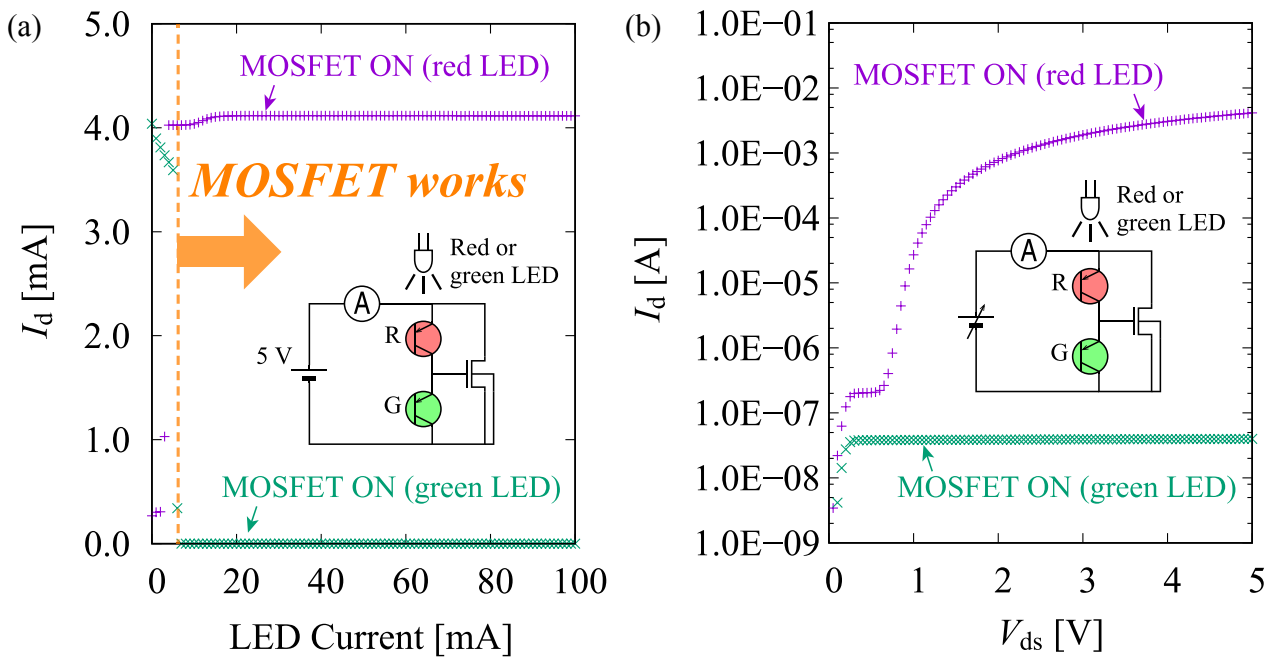

Fig. 3. Drain current characteristics of a single n-MOSFET block against (a) LED current and (b) drain voltage
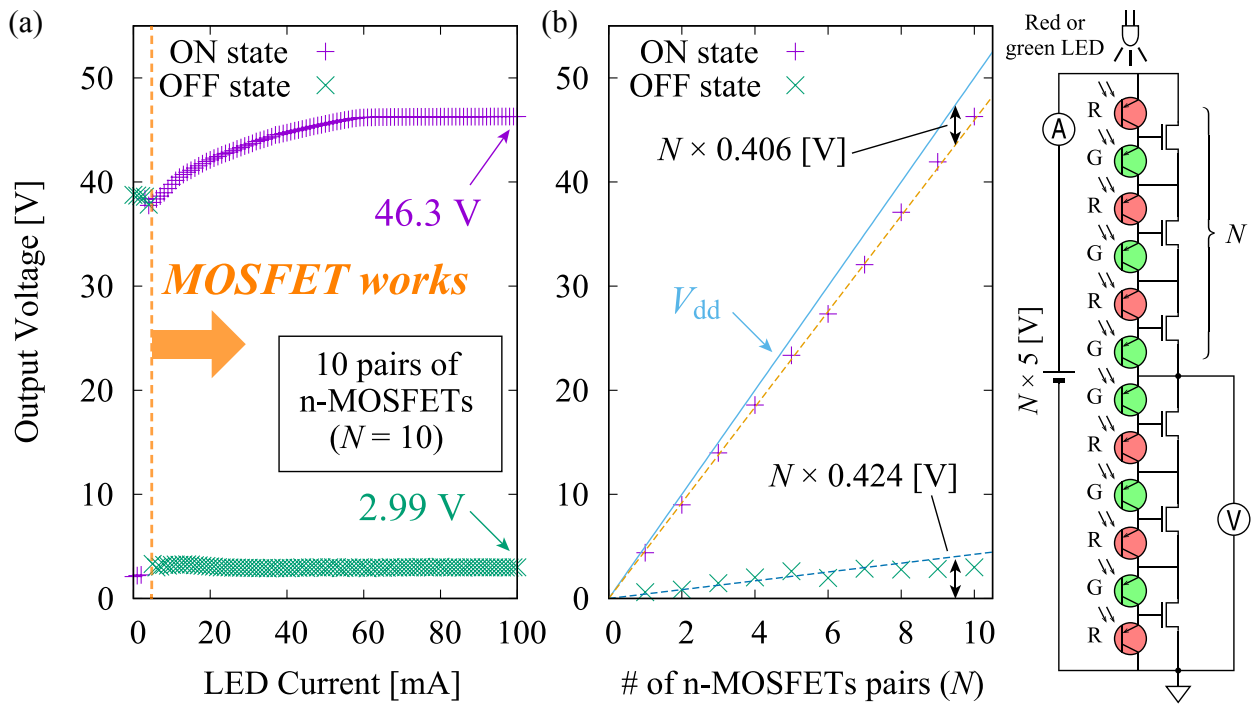

Fig. 4. Output voltage characteristics of the $50 \mathrm{~V}$ switch against (a) LED current and (b) the number of n-MOSFET pairs

maximum value of $46.3 \mathrm{~V}$ at high illumination $\left(I_{\mathrm{LED}}>60 \mathrm{~mA}\right.$, which is over 12 times of threshold value), and (3) 'low-side' voltage stays constant (at $2.99 \mathrm{~V}$ ), independent of incident light power. These results can be well explained by single building block measurement as shown in the Fig. 3. On one hand, under red light (ON state), the on-current depends on $V_{\mathrm{ds}}$ and the typical value sets of $\left(I_{\mathrm{d}}, V_{\mathrm{ds}}\right)$ are $(203 \mathrm{nA}, 0.4 \mathrm{~V})$ and $(4.13 \mathrm{~mA}, 5 \mathrm{~V})$, respectively. The dependence of the FET oncurrent under red light is because the gate and drain are tied through the 'red' phototransistor, therefore the FET works under diode connection: $I_{\mathrm{d}}=\mu C_{\mathrm{ox}} W /$ $L\left(V_{\mathrm{gs}}-V_{\mathrm{th}}\right)^{2} / 2$. On the other hand, under green light (OFF state), the off-current stays constant, approximately around $30 \mathrm{nA}$ independent of $V_{\mathrm{ds}}$. The output voltage under no load is obtained as the result of balancing between the upper circuit that 'pulls-up' the circuit towards $V_{\mathrm{dd}}(50 \mathrm{~V})$, and the lower circuit that 'pulls-down' the circuit towards GND $(0 \mathrm{~V})$. 
In the measured case, there were through current of $247 \mathrm{nA}$ under red (ON) light and of $29.7 \mathrm{nA}$ under green (OFF) light. According to the I-V characteristics in the Fig. 3(b), voltage drop across an $\mathrm{ON}$ transistor was $0.4 \mathrm{~V}$ for $I_{\mathrm{d}} \approx 200 \mathrm{nA}$, resulting $0.4 \times 10=4 \mathrm{~V}$ of voltage drop through $\mathrm{ON}$ transistors, thus $V_{\text {out }} \approx 46 \mathrm{~V}$. Accordingly, under green (OFF) light the 'pull-down' transistors were weakly polarized by $29.7 \mathrm{nA}$ of off-current, that made approximately $0.4 \mathrm{~V}$ of voltage drop at each stage. The above-mentioned analysis is in a good accordance with voltages measurement at each transistor stage, under ON and OFF condition, as shown in the Fig. 4(b). The fact that voltage drop is proportional to the number of transistor proves the voltage scalability of the proposed device.

The equivalent series resistance can also be calculated by single stage I-V characteristics. The equivalent output resistance for measured transistor was $1 \mathrm{k} \Omega$ $(=4 \mathrm{~V} / 4 \mathrm{~mA})$, and is multiplied by series number $N(=10)$, yielding $10 \mathrm{k} \Omega$. Taking the typical value of MEMS electrostatic actuator into account (around $10 \mathrm{pF}$ ), the time constant of charging is around $100 \mathrm{~ns}$, which is sufficient for typical MEMS operation.

\section{Conclusion}

We proposed a high-voltage switch driven by wavelength-modulated light and demonstrated $0-50 \mathrm{~V}$ switching by 20 -series switch. This paper showed that using an optical control can achieve a scalable high voltage switch by a standard CMOS process. Although a diode connection structure of the switch causes a gap of at least $N \times 0.4 \mathrm{~V}$ between the ideal and real output voltages, the switch is applicable to the operation of electrostatic actuators, which requires high-voltage switching.

\section{Acknowledgments}

The CAD patterns were designed with Cadence Virtuoso, accessible through the University of Tokyo, VLSI Design and Education Center (VDEC)'s academic program and were directly patterned by using the VDEC's 8-inch EB writer F5112+VD01 donated by Advantest Corp. Phenitec Semiconductor Corp. is acknowledged for their very kind assistance in their CMOS fabrication service on SOI wafers via VDEC. The post-process was performed using the open facilities maintained by MEXT's Nanotechnology Platform Program. The work was partially supported by Japan Society for the Promotion of Science (JSPS) KAKENHI Grant Number 23686053 and 16H04345. 